\title{
Impact of asymmetric martensite and austenite nucleation and growth behavior on the phase stability and hysteresis of freestanding shape-memory nanoparticles
}

\author{
Won-Seok Ko, ${ }^{1,2, *}$ Blazej Grabowski, ${ }^{2}$ and Jörg Neugebauer ${ }^{2}$ \\ ${ }^{1}$ School of Materials Science and Engineering, University of Ulsan, Ulsan 44610, Republic of Korea \\ ${ }^{2}$ Max-Planck-Institut für Eisenforschung GmbH, Max-Planck-Str. 1, 40237 Düsseldorf, Germany
}

(Received 3 November 2017; revised manuscript received 4 February 2018; published 20 March 2018)

\begin{abstract}
Martensitic transformations in nanoscaled shape-memory alloys exhibit characteristic features absent for the bulk counterparts. Detailed understanding is required for applications in micro- and nanoelectromechanical systems, and experimental limitations render atomistic simulation an important complementary approach. Using a recently developed, accurate potential we investigate the phase transformation in freestanding $\mathrm{Ni}-\mathrm{Ti}$ shape-memory nanoparticles with molecular-dynamics simulations. The results confirm that the decrease in the transformation temperature with decreasing particle size is correlated with an overstabilization of the austenitic surface energy over the martensitic surface energy. However, a detailed atomistic analysis of the nucleation and growth behavior reveals an unexpected difference in the mechanisms determining the austenite finish and martensite start temperature. While the austenite finish temperature is directly affected by a contribution of the surface energy difference, the martensite start temperature is mostly affected by the transformation strain, contrary to general expectations. This insight not only explains the reduced transformation temperature but also the reduced thermal hysteresis in freestanding nanoparticles.
\end{abstract}

DOI: 10.1103/PhysRevMaterials.2.030601

Diffusionless martensitic transformations have received great interest owing to their broad academic and technological relevance. Shape-memory alloys (SMAs) are a representative class of materials utilizing the martensitic transformation [1]. SMAs are widely used in many applications by virtue of their shape-memory and superelasticity behavior, which can be controlled by reversible temperature- and stress-induced martensitic transformations, respectively [1]. As applications of SMAs recently entered the arena of smart materials for microand nanoelectromechanical systems [2-6] and nanocomposite materials with exceptional mechanical properties [7-9], the distinctive characteristics of martensitic phase transformations at the nanoscale have attracted particular attention.

A common characteristic of phase transformations in nanoscale SMAs is an overstabilization of the austenite over the martensite phase [2]. If the system size approaches the nanometer scale, a decrease of the transformation temperature is observed for various kinds of SMAs [2], e.g., nanocrystalline SMAs [10], shape-memory nanoprecipitates embedded in a stiff nontransforming matrix [11], and freestanding shapememory nanoparticles [12]. In the case of nanoscale SMAs with external constraints such as nanocrystalline SMAs and shape-memory nanoprecipitates, the suppression of the phase transformation was explained by an increasing importance of the strain energy owing to mechanical constraints $[2,13]$. Recent molecular-dynamics (MD) simulations on nanocrystalline SMAs [14] and shape-memory nanoprecipitates [15] verified such a scenario.

In the case of freestanding nanoparticles, the situation is different since the external mechanical constraints are lacking.

\footnotetext{
*Corresponding author: wonsko@ulsan.ac.kr
}

The surface energy has been suggested to account for the reported inhibition of the phase transformation [2], while the contribution of strain energy has been neglected. As experiments on shape-memory nanoparticles face difficulties in synthesizing nanoparticles of uniform size, MD simulations have been initiated [16,17] and they seem to support the scenario that the surface energy governs the transformation temperature of freestanding nanoparticles. However, a detailed analysis of the correlation between the evolution of microstructure (nucleation and growth of austenite and martensite) and the resultant characteristic of the phase transformation (transformation temperatures and thermal hysteresis) is still missing. Moreover, the twinning behavior, critical for phase transformations in SMAs, was either not investigated [16] or not analyzed in relation to the transformation characteristics [17].

Here we provide a comprehensive understanding of the phase transformation in freestanding shape-memory nanoparticles by analyzing atomistic details of the nucleation and growth behavior derived from MD simulations. Surprisingly, in contrast to expectations [2,16,17], the strain energy is an important factor, at least for the austenite to martensite transformation. We not only explain the reduced transformation temperature but also the reduced thermal hysteresis in freestanding nanoparticles.

MD simulations were performed using the LAMMPS code [18] with a recently developed second-nearest-neighbor modified embedded-atom method potential for the Ni-Ti binary system [19]. Importantly, this potential accurately reproduces the temperature- and stress-induced phase transformation between B2 austenite and B19' martensite. All MD simulations were performed with a time step of 2 fs utilizing the Nosé-Hoover thermostat $[20,21]$. Freestanding spherical equiatomic NiTi 


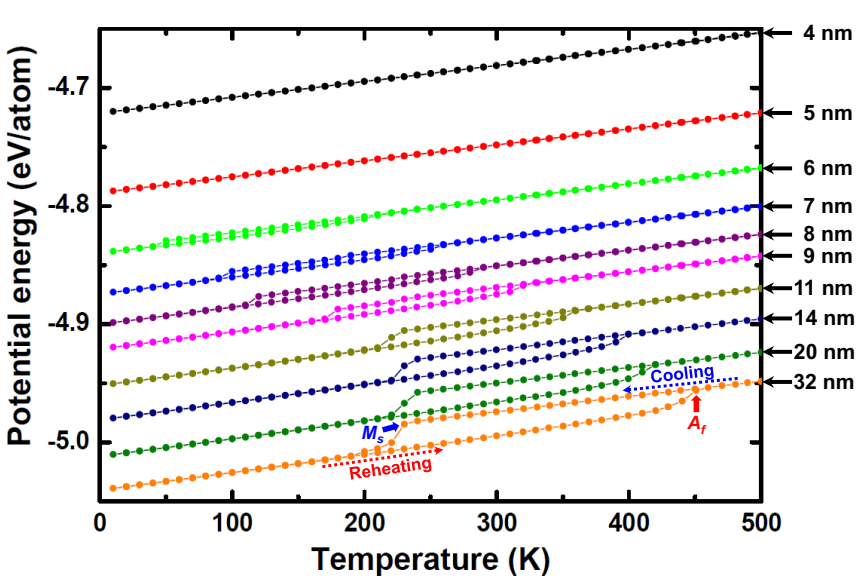

(a)

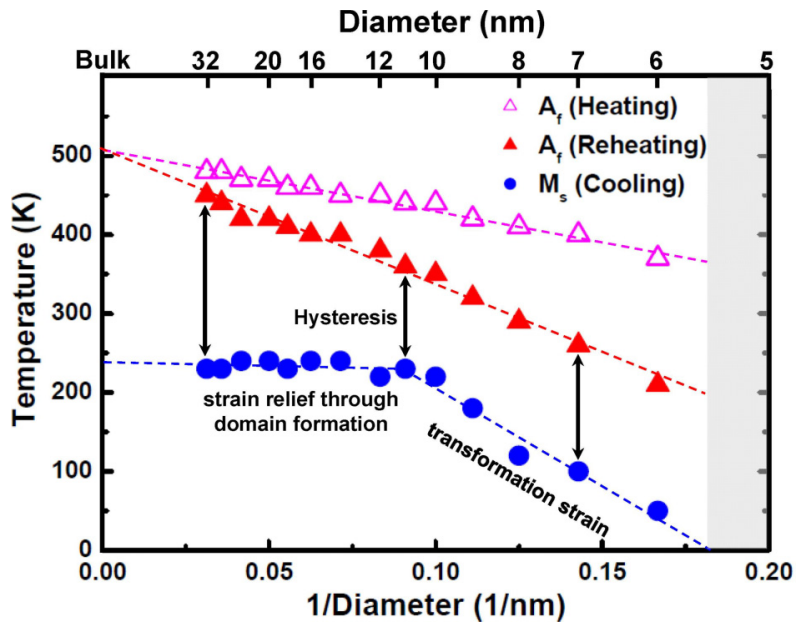

(b)

FIG. 1. (a) Potential-energy dependence of freestanding NiTi particles with various diameters during cooling and reheating. The martensite start $\left(M_{s}\right)$ and austenite finish $\left(A_{f}\right)$ temperatures of the 32-nm particle are indicated by the arrows. (b) Size dependence of the $M_{s}$ and $A_{f}$ temperatures obtained from the curves in (a), and the $A_{f}$ temperatures obtained from the heating process. The gray shading indicates the region where the transformation is suppressed.

nanoparticles with different diameters $(D=4-32 \mathrm{~nm})$ were prepared. Each simulation cell was subjected to an energy minimization process and equilibrated at $500 \mathrm{~K}$. Thermal cycling was then applied to induce the phase transformation with cooling and heating rates of $\pm 0.5 \mathrm{~K} / \mathrm{ps}$. In order to identify the phase transformation event, atomic configurations were analyzed based on an adaptive cutoff common-neighbor analysis (AC-CNA) algorithm [22] as implemented in the OVITO program [23].

Figure 1(a) shows the potential energy dependence (a good indicator of the transformation) during cooling and subsequent reheating for a subset of the nanoparticles. Coming from high temperatures (500 to $240 \mathrm{~K}$ ), all particles maintain the austenite phase, showing a linearly decreasing potential energy when temperature is reduced. At around $240 \mathrm{~K}$, the larger particles show a sudden jump downward in the potential energy representing the transformation. The temperature corresponding to the initial point of the jump is recorded as the martensite start temperature $\left(M_{S}\right)$. With decreasing particle size the transformation temperature decreases and the jump in the energy becomes less pronounced, ceasing for the 4- and 5-nm particles. The decrease in the potential energy due to the transformation can be related to the structural energy difference between the parent (B2 austenite) and transformed phase (B19' martensite). This is consistent with density-functionaltheory calculations $[19,24,25]$, which reported a more negative (stable) structural energy for the B19' martensite phase than for B2 austenite. Overall, the potential energy per atom of a nanoparticle increases with decreasing particle size due to an increase in the surface area to volume ratio and the corresponding relative (i.e., scaled by the total number of atoms in the nanoparticle) increase in broken bonds near the surface area.

Figure 2 shows representative cross sections of particles with various sizes for the initial state $(500 \mathrm{~K})$, after cooling $(10 \mathrm{~K})$, and after reheating. The B2 austenite phase is represented by blue atoms and the B19' martensite phase by red and gray atoms. At the initial temperature of $500 \mathrm{~K}$, each particle consists of pure austenite (some atoms appear in gray due to displacements originating from thermal vibrations and due to surface relaxation). After cooling to $10 \mathrm{~K}$, most particles are composed of the martensite phase with a certain amount of retained austenite in the surface region. Particles with diameters larger than $5 \mathrm{~nm}$ indicate a clear phase transformation while the one with a diameter of $4 \mathrm{~nm}$ does not show any signal of the transformation. The particle with a diameter of $5 \mathrm{~nm}$ shows an intermediate transformation behavior. While the CNA pattern indicates a certain amount of the martensite phase, the change in the potential energy [Fig. 1(a)] is too small to be observed.

Figure 1(a) also displays the potential energy dependence during the reheating process. The energy increases linearly with temperature far above the $M_{s}$ temperature (hysteresis), until a sudden jump upward is observed (around $400 \mathrm{~K}$ for the larger particles). This jump corresponds to the occurrence of the martensite to austenite transformation, and the corresponding temperature is recorded as the austenite finish $\left(A_{f}\right)$ temperature. Above the $A_{f}$ temperature, a complete match between the potential energy dependencies of cooling and reheating is observed. This indicates a complete recovery of the initial B2 austenite phase after reheating, as also confirmed by the atomic configurations in Fig. 2.

The resultant particle-size dependence of the transformation temperature is summarized in Fig. 1(b). For the $A_{f}$ temperature (filled red triangles), there is a clear correlation with the reciprocal diameter $\left(T \propto D^{-1}\right)$ revealing an overstabilization of the austenite phase in smaller particles. A similar trend was reported by previous MD studies for $\mathrm{NiTi}$ [16] and $\mathrm{NiAl}$ [17] SMAs. The correlation can be understood by an analogy to the solid-liquid transition as described by the well-known GibbsThomson model [26]. The model relates the decrease of the melting point with particle size to an increasing contribution of the surface energy, leading to the same relation as found in the present case, i.e., $T \propto D^{-1}$ [26]. This close analogy with the Gibbs-Thomson description suggests that the surface energy is also a key factor determining the martensite to austenite transformation. Specifically, the difference between the austenite and martensite surface energy should scale with the transformation temperature. From the observed lowering of the transformation temperature with particle size, i.e., with 


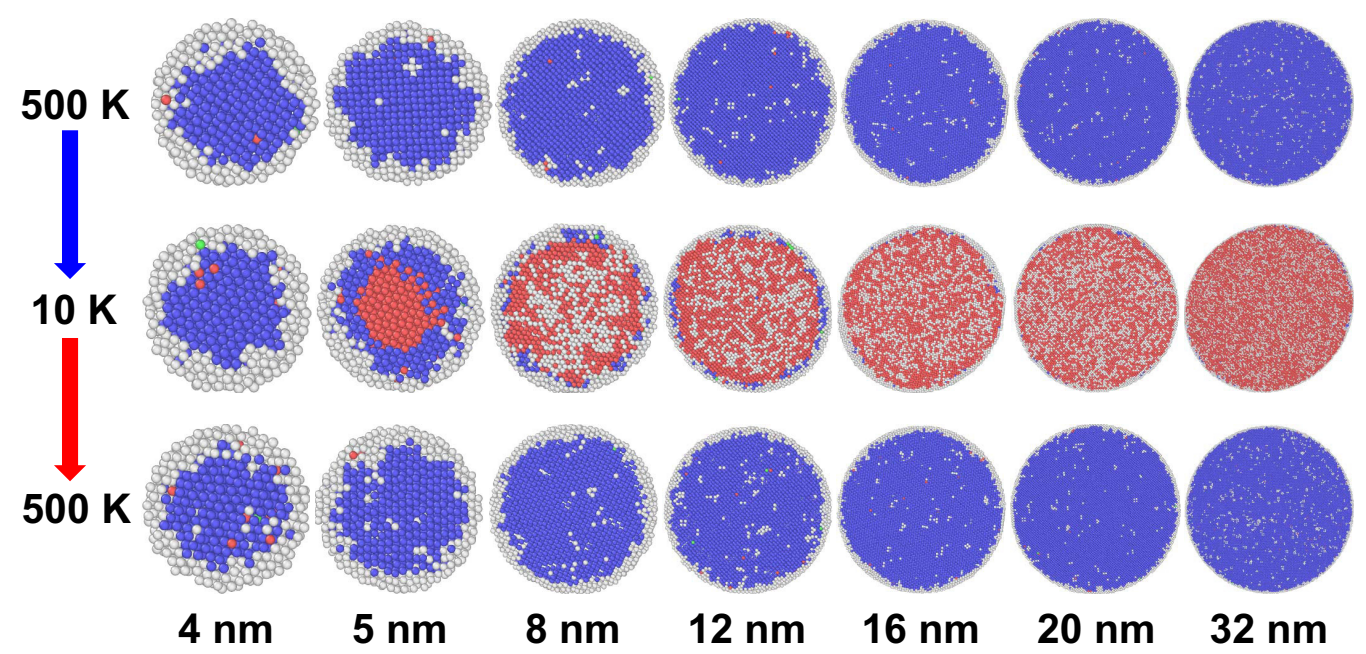

FIG. 2. Cross sections of NiTi particles with various diameters at the initial state (500 K), after cooling (10 K), and after reheating (500 K). The color of the atoms is scaled according to the AC-CNA pattern. The B2 austenite phase is represented by blue atoms and the B19' martensite phase by a mixture of red and gray atoms. Atoms on the free surface appear in gray.

increasing contribution of the surface energy, we thus deduce that the surface of the austenite phase is more stable (has a lower surface energy) than the one of the martensite phase. An overstabilization of the austenite phase for small particles is the consequence.

A direct calculation of the surface Gibbs energies at finite temperatures is challenging for the dynamically unstable austenite phase. We have instead analyzed surface energies at $0 \mathrm{~K}$ prohibiting fully or partially the relaxation of atomic positions to circumvent the dynamical instability. We started with ideal single-phase (i.e., austenite or martensite) spherical nanoparticles and considered two scenarios: (i) all atoms were constrained and (ii) the first few surface layers $(<0.5 \mathrm{~nm})$ were allowed to relax. The surface energies were computed using $\gamma=\left(E_{\text {nano }}-N_{\text {nano }} E_{\text {bulk }}\right) / A$, where $E_{\text {nano }}$ is the total energy of the nanoparticle, $N_{\text {nano }}$ is the number of atoms in the nanoparticle, $E_{\text {bulk }}$ is the energy per atom of the corresponding perfect bulk, and $A$ is the surface area. The resultant fully unrelaxed surface energies of the austenite and martensite phases for the largest particles (32 nm) are 2776 and $2841 \mathrm{~mJ} / \mathrm{m}^{2}$, respectively. The same qualitative result, i.e., lower surface energy for austenite than for martensite, is obtained when the surface layers $(<0.5 \mathrm{~nm})$ are relaxed $(2608$ and $2757 \mathrm{~mJ} / \mathrm{m}^{2}$ ). Thus, the surface energy results are fully consistent with the above expectation.

Atomistic analysis of the nucleation and growth processes further supports the critical influence of the surface energies on the $A_{f}$ temperature. Figure 3 shows representative snapshots of the martensite to austenite transformation for the 28-nm particle during reheating (middle row). The austenite phase (blue) starts growing in the surface region and then toward the center of the particle. This behavior could be a consequence of the lower surface energy of austenite compared to martensite, but in principle also of the fact that the retained austenite in the surface region provides low-energy nucleation sites. In Fig. 3, we therefore present results of additional simulations labeled as "heating" (bottom row). These calculations were started from perfect B19' martensite particles cut from a corresponding bulk (with the same size as the "reheating" simulations). Although this case is hypothetical, it allows us to investigate the impact of the surface-energy difference without the interference of any retained austenite. These idealized simulations show the same behavior, i.e., austenite nucleates at the surface and grows toward the center of the particle. We thus conclude that the reduced nucleation barrier at the surface is caused by the preferred surface energy of the austenite phase.

In Fig. 1(b), the particle-size dependence of the $A_{f}$ temperature for the idealized martensite particles (open pink triangles) is compared to the dependence of $A_{f}$ for the reheated martensite particles (filled red triangles). Extrapolating to an infinite particle diameter (bulk SMA), both dependencies indicate a converged $A_{f}$ temperature $(\approx 510 \mathrm{~K})$ consistent with previous MD results for bulk NiTi [19]. For smaller particle sizes, the increasing difference between the two dependencies implies that the surface condition, i.e., the presence or absence of retained austenite, plays a role in the martensite to austenite transformation. Specifically, the initial surface condition is relevant for the slope of the $A_{f}$ temperature dependence, but the underlying mechanism reflected by the $T \propto D^{-1}$ relation appears to be general.

Our analysis has clearly shown that the surface energy is a key factor for the martensite to austenite transformation. The situation is completely different for the austenite to martensite transformation, i.e., for the $M_{s}$ temperature dependence [Fig. 1(b), filled blue circles]. The $T \propto D^{-1}$ relation no longer applies for particle sizes larger than $\approx 10 \mathrm{~nm}$. At this size a change in the transformation mechanism occurs and we see a constant $M_{s}$ temperature $(\approx 240 \mathrm{~K})$. For larger particles the austenite to martensite transformation cannot be explained solely by the contribution of the surface energy.

To clarify the origin of this transformation behavior, we have investigated the details of the nucleation and growth of the martensite phase. Figure 3 (top row) shows the results for a $28-\mathrm{nm}$ particle, revealing that the nucleation and growth behavior of martensite are remarkably different from those of austenite. The nucleation of the martensite phase (blue) starts 


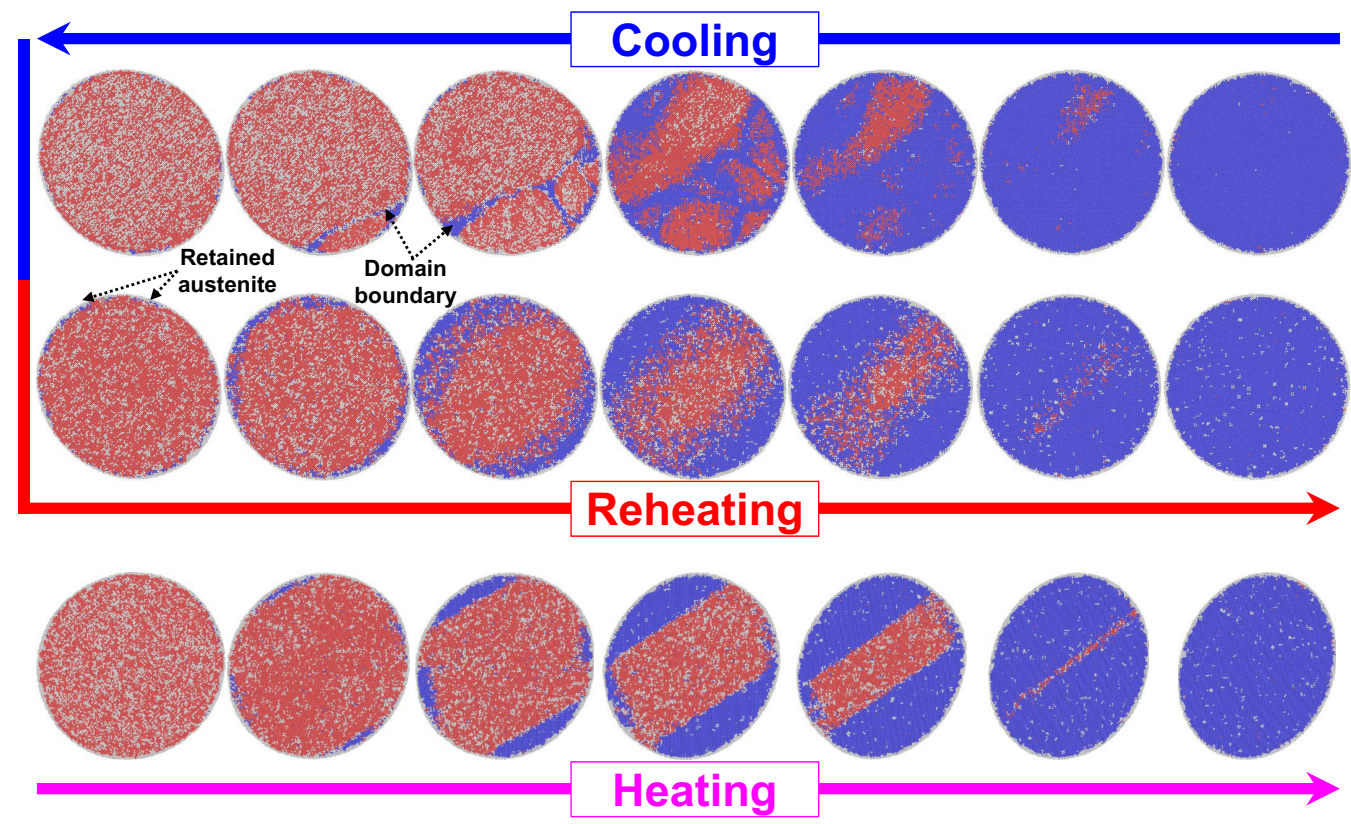

FIG. 3. Cross sections of a NiTi particle $(28 \mathrm{~nm})$ during cooling, reheating, and heating. Color coding as in Fig. 2.

inside of the particle, as opposed to the nucleation of the austenite phase which starts exclusively at the surface. Different martensite nuclei appear at statistically varying places inside of the particle, and they grow to become-for a short time-a network of domains which disappears upon further cooling. Thus, the austenite to martensite transformation proceeds by homogeneous nucleation without the aid of the surface heterogeneity.

The tendency of the martensitic nuclei to avoid the surface region can be explained by the surface-energy difference discussed above. Because the austenite surface is more stable than the martensite surface, martensite nuclei prefer to form inside of the particle rather than at the surface. However, one should not infer from this behavior that the $M_{s}$ temperature is directly determined by the surface-energy difference. Since the martensite nuclei form at some distance away from the surface, the surface energy cannot be a directly contributing factor.

The peculiar $M_{s}$ temperature dependence can be understood by analyzing the strain evolution during the transformation. In the theory of solid-solid phase transformations, the formation of a nucleus is understood to require the deformation of the surrounding matrix and the resulting strain is referred to as transformation strain. The transformation strain can oppose the formation of nuclei if the surrounding matrix exerts mechanical constraints. This is not the case for the martensite to austenite transformation where the nuclei form at the surface, allowing for a direct strain relief through the surface. For the austenite to martensite transformation where the nuclei do not form directly at the surface, corresponding strain relief is limited.

An alternative strain-relief mechanism was reported for confined systems. A significant amount of transformation strain can be relieved by a unique martensite structure with multiple twin variants called "herringbone structure" [27]. This structure was confirmed by MD studies on shape-memory nanoprecipitates [15] and nanocrystalline [14] NiTi SMAs. So far, freestanding particles have been regarded as free of the formation of multiple variants [2]. This expectation seems to be supported by our final atomic structures after completion of the martensitic transformation (Fig. 2, middle row) which do not show any multiple variants.

However, the final atomic structures are not representative of the transformation kinetics. It is necessary to investigate the evolution of the microstructure during the transformation. As mentioned above, the snapshots of the cooling process for the 28-nm particle (Fig. 3; top row) reveal clearly that, at the initial stage of the transformation, multiple variants of martensite nuclei form at different locations. They grow to form a network of domains separated by domain boundaries. Even though these boundaries disappear shortly after their formation into the free surface and are absent when the transformation is completed, they provide a viable strain-relief mechanism for freestanding particles and affect the $M_{s}$ temperature which represents the very first stage of the nucleation and growth process.

With this understanding, we can explain the constant $M_{s}$ temperature $(\approx 240 \mathrm{~K})$ observed for particles with diameters $D>10 \mathrm{~nm}$. The formation of multiple variants during the austenite to martensite transformation reduces the strain energy, but the accompanying domain boundaries increase the interfacial energy. The system balances the strain and interfacial energy such that the sum of both contributions to the total free energy remains constant. Thus, as long as multiple variants are able to form, the nucleation barrier does not significantly depend on the size of the particle, resulting in the constant $M_{s}$ temperature dependence. This is supported by Fig. 4, which displays the structural evolution of particles with various diameters during the cooling process. All particles with diameters larger than $10 \mathrm{~nm}$, i.e., the ones with the same $M_{s}$ temperature, involve the formation of multiple variants.

According to the present results (Fig. 4), a diameter of $10 \mathrm{~nm}$ represents a critical particle size that distinguishes particles 


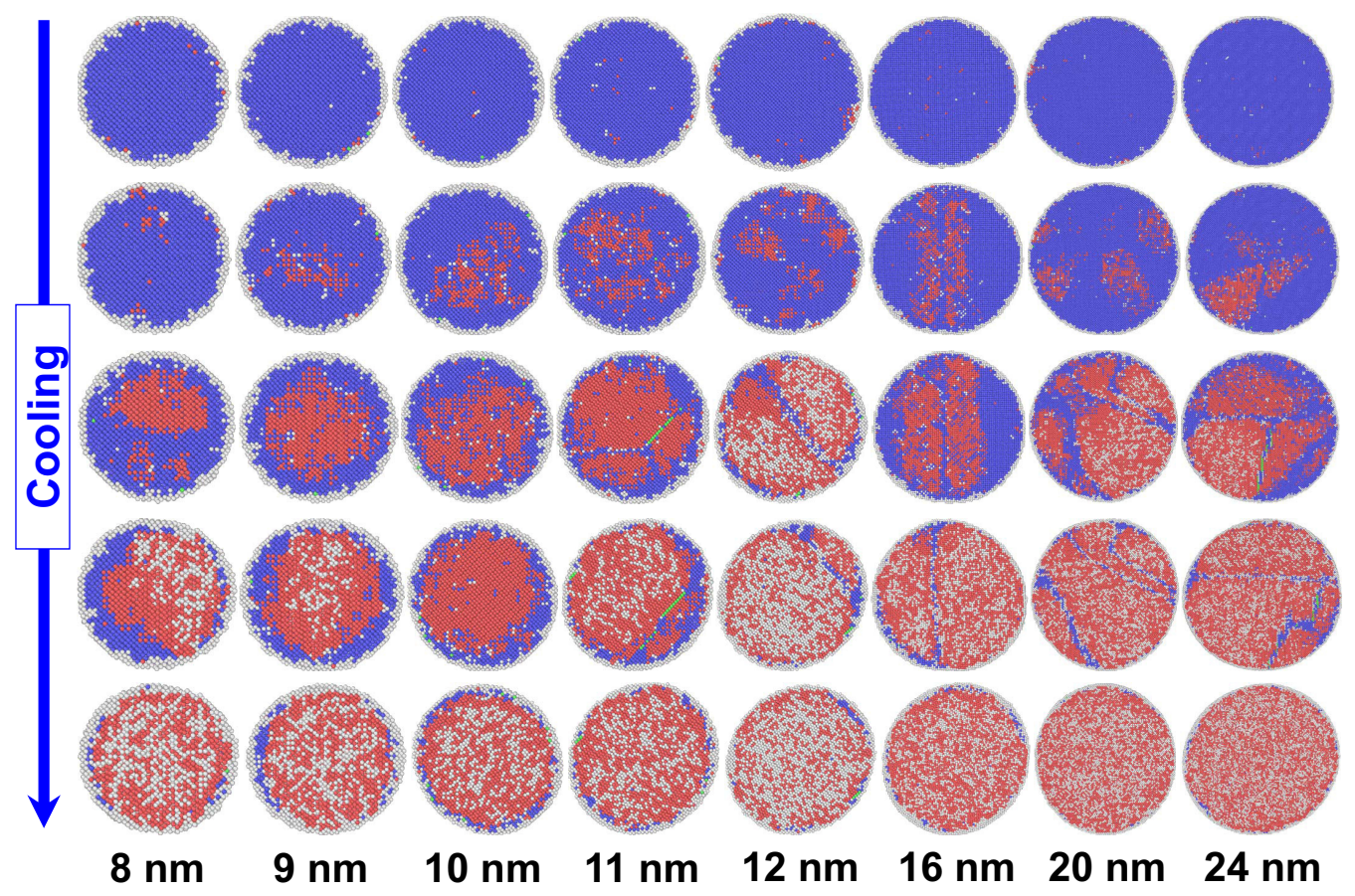

FIG. 4. Cross sections of NiTi particles with various diameters during the austenite to martensite transformation. Color coding as in Fig. 2.

with and without the formation of multiple variants. Particles with diameters smaller than or equal to $10 \mathrm{~nm}$ do not involve the formation of multiple variants. Therefore, at these particle sizes, the transformation strain cannot be relieved anymore and contributes to the nucleation barrier resulting in the decrease of the $M_{s}$ temperature with particle size. In general, the critical particle size will depend on the material system, in particular on the elastic and defect properties of the austenite and martensite phases. However, we expect that the underlying mechanism of the phase transformation is generic and common to other SMAs.

We have revealed that the $A_{f}$ and $M_{s}$ temperatures are determined by fundamentally different nucleation and growth mechanisms. The $A_{f}$ temperature decreases proportionally to the inverse of the particle size, while the $M_{s}$ temperature-in contrast to previous expectations-maintains a steady value until the multiple domain structure is unsustainable. The hysteresis, i.e., the difference between the $A_{f}$ and the $M_{s}$ temperatures, decreases with decreasing particle size as long as
$D>10 \mathrm{~nm}$, in consistency with previous experimental results on AuCd SMA [12]. The present simulations successfully explain the atomistic origin of such a behavior. Moreover, we predict that if the size of the particle becomes smaller than the critical value $(D<10 \mathrm{~nm})$, the hysteresis dependence will drastically differ, showing only a small change with particle size.

Funding by the Deutsche Forschungsgemeinschaft (Grant No. SPP 1568) and by the European Research Council (ERC) under the European Union's Horizon 2020 research and innovation program (Grant Agreement No. 639211) is gratefully acknowledged. W.-S.K. acknowledges the National Research Foundation of Korea (NRF) grant funded by the Korea government (MSIP) (Grant No. NRF-2017R1C1B5015038). W.-S.K. also acknowledges the supercomputing resources including technical support from the National Institute of Supercomputing and Network/Korea Institute of Science and Technology Information (Grant No. KSC-2016-S1-0015).
[1] K. Otsuka and X. Ren, Prog. Mater. Sci. 50, 511 (2005).

[2] T. Waitz, K. Tsuchiya, T. Antretter, and F. D. Fischer, MRS Bull. 34, 814 (2009).

[3] J. S. Juan, M. L. No, and C. A. Schuh, Nat. Nanotechnol. 4, 415 (2009).

[4] J. R. Greer and J. T. M. De Hosson, Prog. Mater. Sci. 56, 654 (2011).

[5] A. Lai, Z. Du, C. L. Gan, and C. A. Schuh, Science 341, 1505 (2013).

[6] J. F. Gómez-Cortés, M. L. Nó, I. López-Ferreño, J. HernándezSaz, S. I. Molina, A. Chuvilin, and J. M. San Juan, Nat. Nanotechnol. 12, 790 (2017).
[7] D. C. Hofmann, Science 329, 1294 (2010).

[8] S. Pauly, S. Gorantla, G. Wang, U. Kühn, and J. Eckert, Nat. Mater. 9, 473 (2010).

[9] S. Hao et al., Science 339, 1191 (2013).

[10] T. Waitz, T. Antretter, F. D. Fischer, N. K. Simha, and H. P. Karnthaler, J. Mech. Phys. Solids 55, 419 (2007).

[11] T. Waitz and H. P. Karnthaler, Acta Mater. 52, 5461 (2004).

[12] C. Frommen, G. Wilde, and H. Rösner, J. Alloys Compd. 377, 232 (2004).

[13] A. Ahadi and Q. Sun, Acta Mater. 90, 272 (2015).

[14] W.-S. Ko, S. B. Maisel, B. Grabowski, J. B. Jeon, and J. Neugebauer, Acta Mater. 123, 90 (2017). 
[15] S. B. Maisel, W. S. Ko, J. L. Zhang, B. Grabowski, and J. Neugebauer, Phys. Rev. Mater. 1, 033610 (2017).

[16] D. Mutter and P. Nielaba, Eur. Phys. J. B 84, 109 (2011).

[17] Z. Zhang, X. Ding, J. Deng, J. Cui, J. Sun, T. Suzuki, K. Otsuka, and X. Ren, J. Phys. Chem. C 117, 7895 (2013).

[18] S. Plimpton, J. Comput. Phys. 117, 1 (1995).

[19] W.-S. Ko, B. Grabowski, and J. Neugebauer, Phys. Rev. B 92, 134107 (2015).

[20] S. Nosé, J. Chem. Phys. 81, 511 (1984).

[21] W. G. Hoover, Phys. Rev. A 31, 1695 (1985).
[22] A. Stukowski, Modell. Simul. Mater. Sci. Eng. 20, 045021 (2012).

[23] A. Stukowski, Modell. Simul. Mater. Sci. Eng. 18, 015012 (2010).

[24] X. Huang, G. J. Ackland, and K. M. Rabe, Nat. Mater. 2, 307 (2003).

[25] D. Holec, M. Friák, A. Dlouhý, and J. Neugebauer, Phys. Rev. B 84, 224119 (2011).

[26] Y. Shibuta and T. Suzuki, J. Chem. Phys. 129, 144102 (2008).

[27] T. Waitz, Acta Mater. 53, 2273 (2005). 